\title{
Cancer permeates locally within ontogenetic compartments: clinical evidence and implications for cancer surgery
}

\section{Michael Höckel}

Department of Gynecology, Women's \& Children's Center, University of Leipzig, Liebigstrasse 20 a, 04103 Leipzig, Germany = Tel.: +493419723400 = Fax: +49 3419723409 = michael.hoeckel@uniklinik-leipzig.de

The pathophysiologic process of local tumor spread is regarded as an isotropic infiltration of microscopic extensions of the malignant lesion irrespective of tissue boundaries. By contrast, the ontogenetic compartment theory states that malignant solid tumors are locally confined, for a relatively long phase during their natural course, to a permissive compartment derived from a common primordium in embryonic development. Tumor permeation is isotropic within the permissive ontogenetic compartment, but it is suppressed at the compartment borders. The validity of the ontogenetic compartment theory has been shown for cancer of the rectum and of the female lower genital tract. It is hypothesized that ontogenetic compartment resection, the translation of the theory into cancer surgery, holds a great potential to improve oncologic treatment results.

\section{Current principles \& practice of cancer surgery}

Local spread of an invasive tumor is currently regarded as an isotropic process of tissue infiltration, irrespective of anatomic boundaries (Figure 1A \& B). Malignant solid tumors are thought to permeate locally by invasion of the interstitium, intravasion of lymphatic and venous channels and perineural spread. As a consequence, microscopic or occult disease is expected to precede the macroscopic tumor front. The surgical practice derived from these conceptions is wide tumor excision (i.e., the removal of the malignant tumor with a metrically defined radial margin of microscopically tumor-free tissue) (Figure 1C \& D). However, despite histopathologically confirmed resection with clear margins (R0), recurrences within the surgical treatment field occur in up to $50 \%$ of high-risk cases [1]. Adjuvant radiotherapy may be administered to lower the rate of local relapses. This bimodal therapy increases treatment-related morbidity but may not prolong overall survival $[2,3]$.

Although considered as the fundamental principle in current cancer surgery the relevance of margin width for the clinical practice is vague at best. Whereas for breast cancer older studies found a positive correlation, newer data from large tumor cohorts could not confirm that local control can be improved by increasing tumor-free margins [4]. A prospective randomized trial comparing radical hysterectomy techniques resecting more or less paracervical tissue stated no influence on local control of cervical cancer [5]. With surgery for vulvar cancer, a critical margin width described in previous investigations [6], could not be reproduced by a recent study of the same design and patient number [7]. The occurrence of a significant rate of local relapses, despite perfectly performed surgery and the missing robustness of margin width to predict local recurrences, question the current pathophysiological model of local tumor permeation.

\section{Ontogenetic anatomy}

The term 'ontogenetic anatomy' is suggested for the systematics of mapping compartments in the human body defined by embryonic development as distinct from functional units, the subject of traditional clinical anatomy [8-12]. The existence of developmental compartments that are presented during ontogenesis were first demonstrated in the wings of Drosophila in 1973 [13]. Developmental compartments are characterized by the fact that proliferating and migrating cells do not mix at their boundaries [14]. During tissue growth and differentiation, the compartment boundaries are maintained and subcompartments with additional demarcation areas are formed. 'Ontogenetic compartments' are differentiated tissue entities derived from distinct precursor tissues, the primordia or anlagen. In the context of ontogenetic anatomy, an anlage describes the earliest discernable epithelium-mesenchyme complex in the embryo with morphogenetic determination. The ontogenetic compartment developing from each anlage is morphologically invariable and may consist of functional tissue and tissues

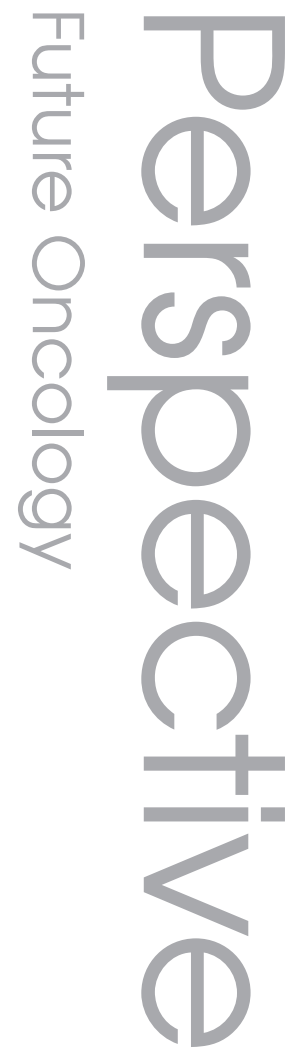

\section{Keywords}

- cancer surgery

- compartment = embryology

- ontogenetic anatomy

- tumor invasion

\section{Future Medicine "arto}




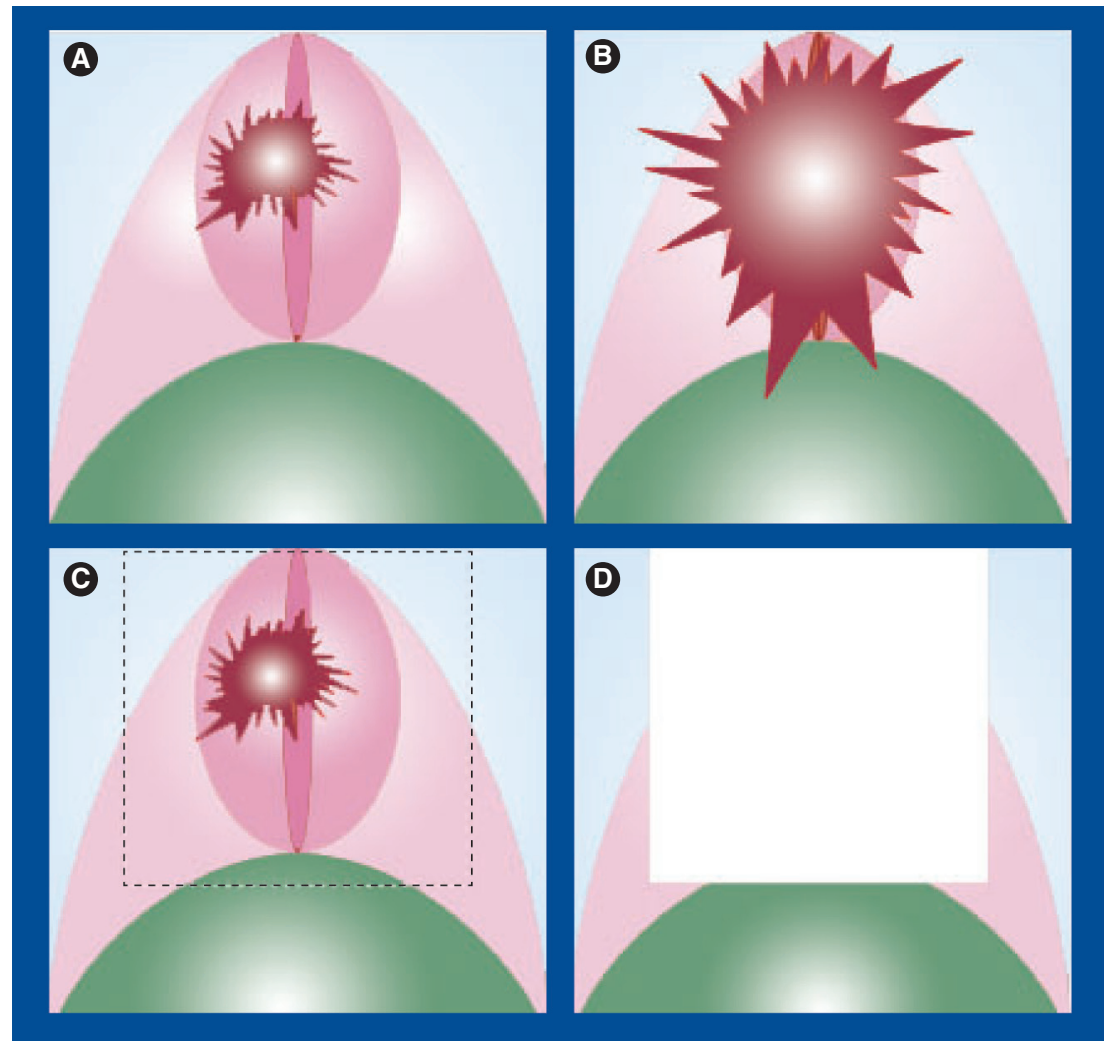

Figure 1. Current concept of local tumor spread guiding cancer surgery. The malignant tumor (red) is considered to permeate isotropically by infiltration with microscopic tumor extensions irrespective of tissue boundaries (A \& B). Wide excision resects the tumor with a metrically defined enveloping margin of tumor-free tissue (C \& D).
- The deep urogenital sinus (UGS)-vaginal plate complex

- The superficial UGS-genital folds and tubercle complex

The mature tissues differentiated from these anlagen form pelvic tissue compartments, which contain parts of the functional genital tract, but also parts of other pelvic functional units and tissue without apparent function. TaBLE 1 provides an overview of the corresponding primordia, the ontogenetic compartments and their relations to the female genital tract. The difference between functional and ontogenetic anatomy is demonstrated with the female perineum where it becomes evident just by inspection (FIgUre 2). Functional anatomy considers the labia majora as part of the vulva, but the anus as a different functional unit. The midperineum located in between is arbitrarily divided into an upper part belonging to the urogenital region and a lower part related to the anal region. Ontogenetically, the labia majora are different from the other parts of the vulva as they are developed from the dorsolateral perineal complex, a precursor tissue derived from the sacral somites. However, the midperineum and the ventral segment of the anus stem from the superficial UGS complex and are ontogenetically integral to the vulva.

with no known or apparent physiologic function. A functional anatomic unit (i.e., a specified tissue or an organ) can be established from more than one anlage and may therefore represent multiple ontogenetic compartments. Conversely, an anlage may develop into more than one functional unit or a part of those. The borders between different ontogenetic compartments can be identified at certain sites in the adult body owing to surface lamellae enveloping the ontogenetic compartment but may be blurred by dense tissue adherences, focal fusion zones or epithelial coverage at other sites. Certain ontogenetic compartments can be visualized by high-resolution MRI [9].

The concept of ontogenetic anatomy, compared with functional anatomy, is described here for the lower female genital tract as an example. In the 8-week embryo, three primordial tissue complexes (anlagen) can be morphologically distinguished to be involved in the formation of that body part in the female phenotype $[8,11,12]$. From cranial to caudal, these primordia are:

- The paramesonephric-mesonephric-Müllerian tubercle complex

\section{Theory of tumor permeation within ontogenetic compartments}

Cellular proliferation, migration and functional differentiation within multicellular compartments represent a general principle in embryonic development, tissue repair and regeneration. Although characterized by abnormal cellular proliferation, migration and differentiation, as an evolutionary multicellular biological system, cancer still depends on essential system-immanent features, such as compartmentalization, for its existence.

The ontogenetic compartment theory of cancer permeation states that malignant solid tumors are locally confined for a relatively long phase during their natural course to permissive ontogenetic compartments; tumor permeation is isotropic within the permissive compartment, but it is suppressed at the compartment borders; transgression into adjacent primarily nonpermissive ontogenetic compartments necessitates phenotypical changes occurring rather late in ceded by focal inflammation at the compartment border and is related to the degree of ontogenetic kinship. malignant progression; transgression is pre- 
Table 1. Ontogenetic anatomy of the female lower genital tract.

\begin{tabular}{|c|c|c|}
\hline $\begin{array}{l}\text { Primordial morphogenetic } \\
\text { unit: anlage }\end{array}$ & $\begin{array}{l}\text { Mature morphogenetic unit: } \\
\text { ontogenetic compartment }\end{array}$ & Suggested term \\
\hline $\begin{array}{l}\text { Paramesonephric-mesonephric- } \\
\text { Müllerian tubercle complex }\end{array}$ & $\begin{array}{l}\text { Fallopian tubes (except fimbria) } \\
\text { Mesosalpingia } \\
\text { Uterus } \\
\text { Mesometria }^{\dagger} \\
\text { Vagina except distal part }^{\text {Mesocolpia }^{\dagger}}\end{array}$ & Müllerian compartment \\
\hline $\begin{array}{l}\text { Deep urogenital sinus-vaginal } \\
\text { plate complex }\end{array}$ & $\begin{array}{l}\text { Urethra } \\
\text { Urethrovaginal septum } \\
\text { Distal vagina } \\
\text { Distal rectovaginal septum }\end{array}$ & $\begin{array}{l}\text { Internal urogenital sinus } \\
\text { compartment }\end{array}$ \\
\hline $\begin{array}{l}\text { Superficial urogenital } \\
\text { sinus-genital folds and } \\
\text { tubercle complex }\end{array}$ & $\begin{array}{l}\text { Vulva except labia majora } \\
\text { Meatus urethrae } \\
\text { Midperineum } \\
\text { Ventral segment of anus }\end{array}$ & $\begin{array}{l}\text { External urogenital sinus } \\
\text { compartment }\end{array}$ \\
\hline
\end{tabular}

The ontogenetic compartment theory is illustrated with Figure 3.

So far, the theory has been verified by clinical evidence with various pelvic tumor entities, such as carcinoma of the rectum, uterine cervix, vagina and vulva. Pelvic MRI of 149 consecutive patients with locally advanced cervical cancer showed that $100 \%$ of stage IB, $95 \%$ of stage II and $73 \%$ of stage IIIB tumors were confined to the ontogenetic Müllerian compartment [10]. Cases with equally sized bilateral tumor involvement mirrored the mesenchyme distribution of the Müllerian anlage at the corresponding level within the pelvis. For cervical cancer recurring after primary radiotherapy, MRI-based pelvic tumor landscapes representing tumor growth fields within an unaltered

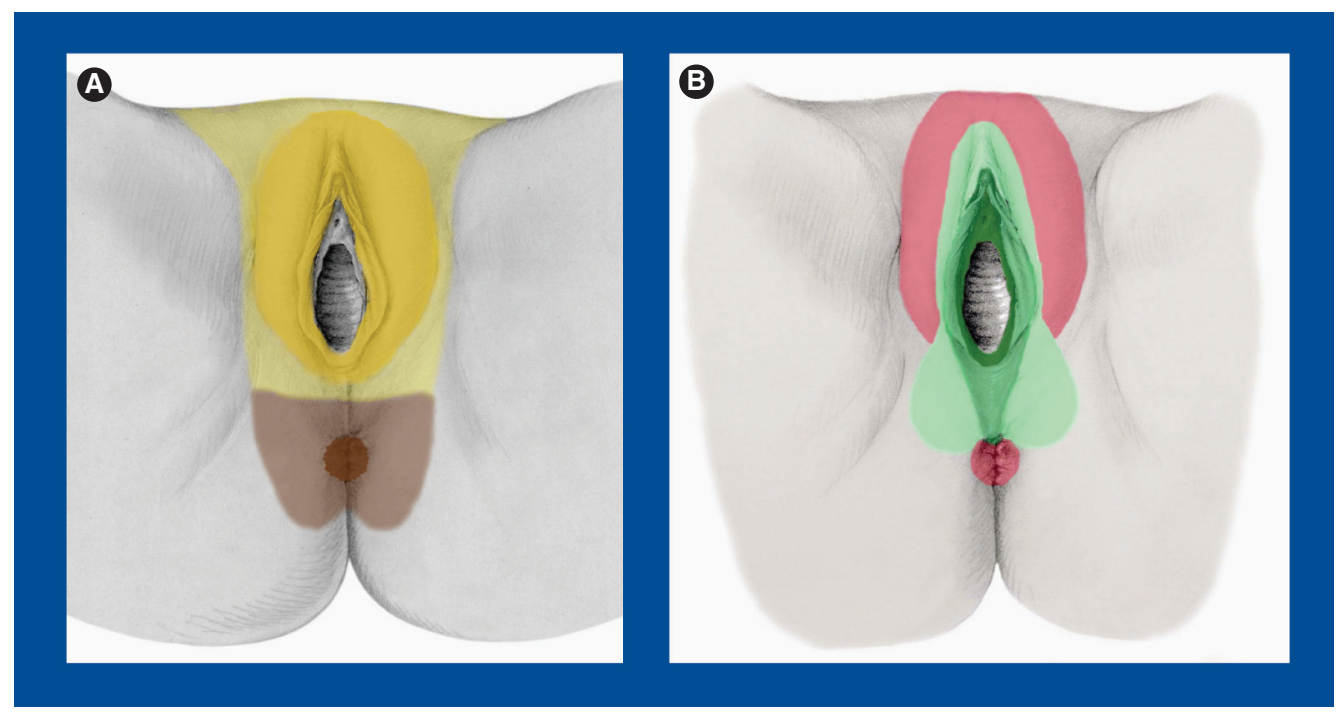

Figure 2. Comparison of functional anatomy and ontogenetic anatomy for the female perineum. (A) Functional anatomy divides the perineum into an urogenital region around the vulva (yellow) and an anal region around the anus (brown). The labia majora are considered as integral part of the vulva and the midperineum is allocated arbitrarily to both regions. (B) Ontogenetically, the labia majora are not derived from the superficial urogenital sinus-genital folds and tubercle complex - the anlage of the vulva - whereas the midperineum and the ventral segment of the anus differentiate from this primordium. The three subcompartments developed from the vulvar anlage are colored green, whereas the perineal derivatives of the dorsolateral perineal complex appear in red. It is apparent by inspection that the ontogenetic vulvar anatomy differs significantly from the functional vulvar anatomy.

(B) is reproduced with permission from [11] @ Elsevier. 


\section{Perspective Höckel}

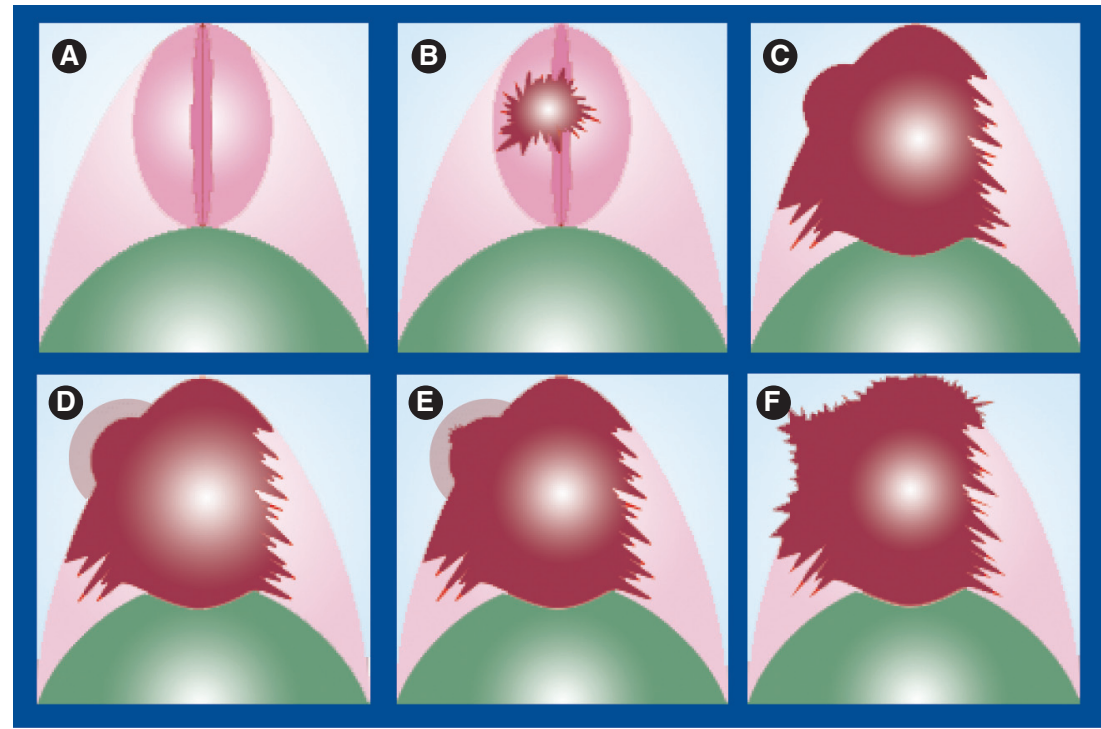

Figure 3. Theory of ontogenetic compartments for local tumor spread.

(A) Shows an ontogenetic compartment (pink) forming a tissue compartment differentiated from a common embryonic anlage. It contains an epithelial subcompartment (shallow ellipse), functional stroma (ellipse) and tissue without apparent function (wings). Adjacent tissues (light blue and green) are derived from different primordia. In the ontogenetic dendrogram, the number of branching points from the compartment of interest to the first common precursor is higher for the green (bottom) than for the light blue (top) tissue. Within the stroma of the compartment, a malignant epithelial tumor permeates isotropically (B). However, compartment borders are functionally tumor suppressive rendering tumor permeation an anisotropic process. The tumor inflates, deforms and destroys the compartment but does not transgress into the adjacent compartment (C).

Transgression necessitates phenotypic changes that usually evolve relatively late in the natural course (i.e., in the locally advanced state). Transgression occurs preferably into the light blue tissue due to the closer ontogenetic kinship. Initial transgression into a neighboring compartment of different embryonic origin is preceded and accompanied by focal inflammation and fibrosis (D \& E). As the transgressing phenotype achieves a growth advantage, multifocal invasion of the adjacent compartment follows (F).

pelvic anatomy, reflect the Müllerian compartment architecture. Likewise, 46 out of 54 consecutive patients with vulvar cancer stages pT1-3 had their macroscopic tumor mass within the external UGS compartment [11]. Also, in accordance with the ontogenetic compartment theory is the observation that, carcinomas of the anterior distal vaginal wall primarily involve the urethra, respectively, the periurethral tissue; however, the rectum is only infiltrated by very advanced tumors of the posterior distal vaginal wall [12]. Both the urethra and the distal vagina belong to the same ontogenetic compartment derived from the deep UGS-vaginal plate complex, whereas the rectum represents a different ontogenetic compartment developed from the hindgut.

The largest amount of clinical data supporting the ontogenetic compartment theory of tumor spread have been published for rectal carcinoma. The rectal compartment differentiated from the embryonic hindgut includes the rectum and the enveloping mesorectum as an ontogenetic compartment [15]. Rectal cancer spread by continuous and discontinuous propagation is confined to the rectal compartment for long phases during malignant progression $[16,17]$. The validity of this principle could also be demonstrated for the distal part of the rectal compartment, which contains the internal anal sphincter, whereas the external anal sphincter belongs to an ontogenetically different tissue compartment derived from the sacral somites [17]. Only rectal carcinomas that are very advanced in malignant progression are able to invade the external anal sphincter (i.e., to transgress into an ontogenetically different compartment).

Evidence for the suppression of tumor growth at the compartment borders is deduced from the fact that, for carcinomas of the uterine cervix and vulva, R0 resection was always histologically confirmed from the dissection sites at the compartment border, despite narrow margins of $\leq 1 \mathrm{~mm}[9,11]$. Although adjuvant radiation was not administered, local recurrence rates were very low $(<5 \%)$ in these cases. Moreover, locally advanced tumors infiltrating the adjacent compartment (stages IIIB and IVA) are relatively well oxygenated, whereas tumors of comparable sizes confined to the primary ontogenetic compartment are severely hypoxic [10]. Therefore, a growth advantage due to better energy balance should result from compartment transgression. Tumor extensions approaching the compartment border should be attracted rather than repelled by the neighboring compartment. As this does not happen for extended phases in the natural disease course, a tumor suppressive function of the compartment border must be concluded from the dynamics of the malignant disease.

The reversal of tissue hypoxia found in cervical carcinomas transgressing into an ontogenetically different compartment also indicates a phenotypic change of the tumor cells as postulated by the theory [10]. Transgression was exclusively observed with large tumor masses and advanced malignant progression was evident by regional and distant metastases both in cervical and vulvar cancer.

A recent analysis of a large prospective hospital registry on resections for colorectal cancer identified tumor fixation as a strong independent prognostic factor for rectal cancer regardless of whether actual invasion of the adherent tissue could be detected [18]. These results support the compartment theory stating that transgression of a tumor into a primarily nonpermissive compartment is associated with advanced malignant progression, and is preceded and accompanied 
by focal inflammation and fibrosis leading to tissue adherence. Likewise, in cervical cancer histologically proven to begin transgression into the bladder was always accompanied by a focal inflammatory adherence at the compartment border [10].

Several observations reported for nonpelvic tumor entities support the compartment theory as well. The finding that early invasive breast cancer is confined to a distinct lobe with its ductal system is in line with the compartment theory as each duct-lobular system of the breast represents an ontogenetic compartment derived from an individual sprout of the mammary epithelial primordium $[19,20]$. For carcinoma of the pancreas, two patterns of perineural spread have been detected to be related to the morphogenesis of this organ by the fusion of two distinct anlagen [21]. Another topographic relation of local tumor spread and embryonic development has been reported for the spatially complex permeation of gliomas of the human insular cortex [22].

By using the number of branching points from a common primordium in the ontogenetic dendrogram, a degree of ontogenetic kinship between organs/tissues can be estimated. For example, the Müllerian derivatives (cervix uteri and proximal vagina) and the bladder share the primitive UGS as common primordium. Two intermediate developmental steps, the formation of the definitive UGS and the separation into a deep and a superficial part, occur before the Müllerian anlage is established. Consequently, the adjacent structures - the cervix, vagina and bladder - have a three-degree ontogenetic kinship. In the ontogenetic dendrogram, the branches of the rectum and proximal vagina unite after four steps in form of the cloaca. These neighboring tissues therefore exhibit a fourdegree ontogenetic kinship. The difference in ontogenetic kinship is inversely associated with the probability that an advanced cervical/vaginal cancer will transgress into the bladder and into the rectum. Of 149 consecutive patients with locally advanced cervical cancer, 34 infiltrated the lower urinary tract but only six instances of rectal infiltration were found [10].

The ontogenetic compartment theory consistently explains the weaknesses of conventional cancer surgery. Local recurrences after surgical cancer treatment may arise from remnants of the ontogenetic compartment remaining in situ after primary treatment harboring or recruiting occult clonogenic tumor cells (Figure 4A \& B). Compartment remnants are left behind with conventional surgery because they may represent tissues without known function or they are integral to other organs. Without being aware of their importance for tumor spread, surgeons are not motivated to resect these tissues.

MRI of female pelvises after standard radical hysterectomy clearly demonstrates remnants of the Müllerian compartment cranial to the vaginal vault. Postsurgical local recurrences of cervical cancer are topographically related to these parts of the Müllerian compartment remaining in situ after radical hysterectomy [9]. The missing robustness of margin width to predict local recurrences following wide tumor excision can be explained by the different impact of a narrow margin at the compartment border as compared with a site within the ontogenetic compartment. With conventional cancer surgery, it is arbitrary whether the resection margin corresponds to the border of the ontogenetic compartment. Therefore, its prognostic relevance is ambiguous (Figure 4C \& D).

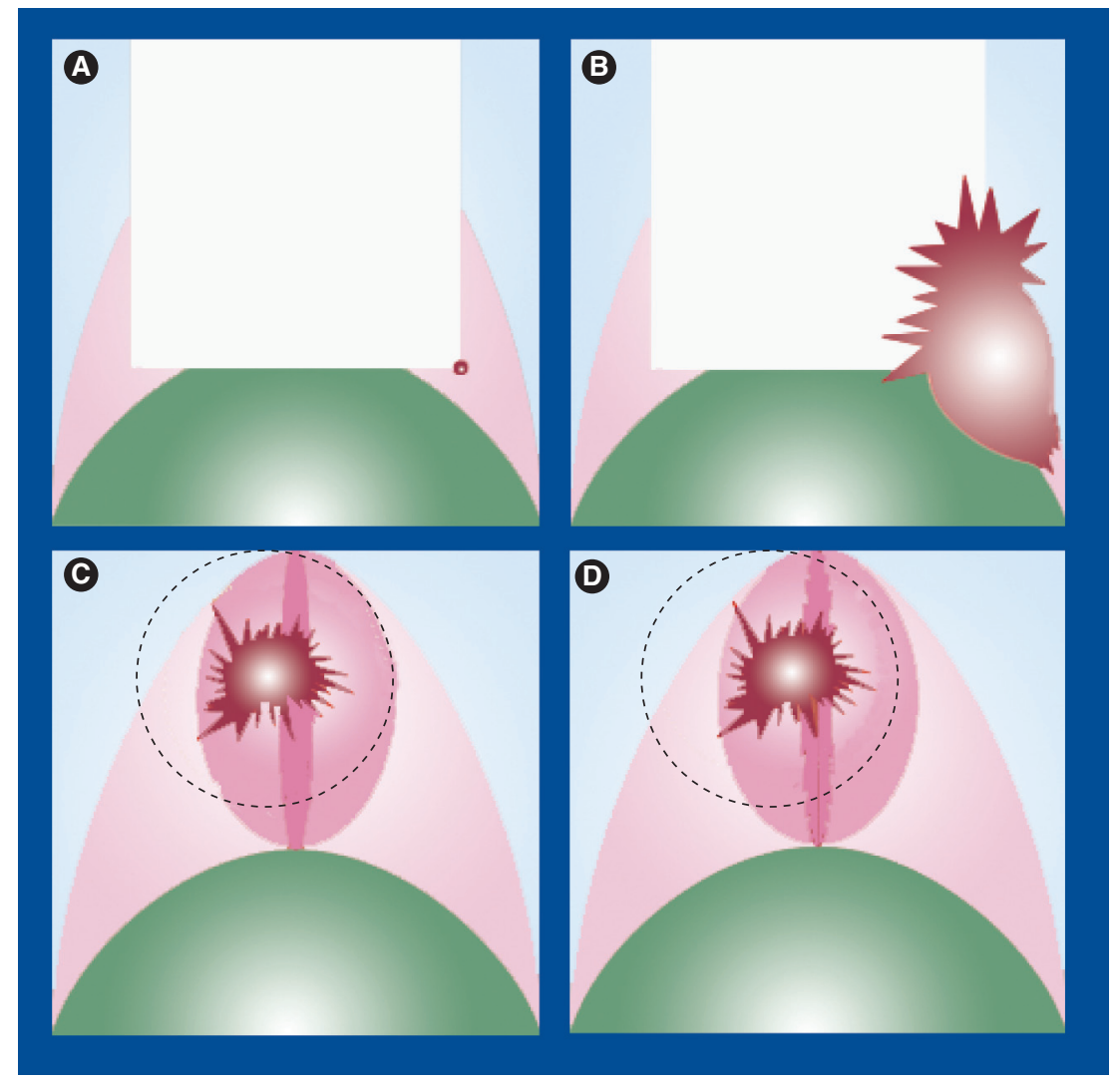

Figure 4. The ontogenetic compartment theory provides explanations for the weaknesses of standard cancer surgery. (A \& B) Local tumor relapses (B) may arise from remnants of the compartment remaining in situ after treatment harboring or recruiting clonogenic tumor cells $(\mathbf{A})$. The missing robustness of margin width, as a predictor of local recurrence, may be due to the differential impact of a narrow margin at the compartment border (C) as compared with a site within the compartment (D). Because, with conventional surgery, the relation of the excision to the compartment border is random, the prognostic relevance of the margin width cannot be robust. Also see legends for Figures 1 \& 3 . 


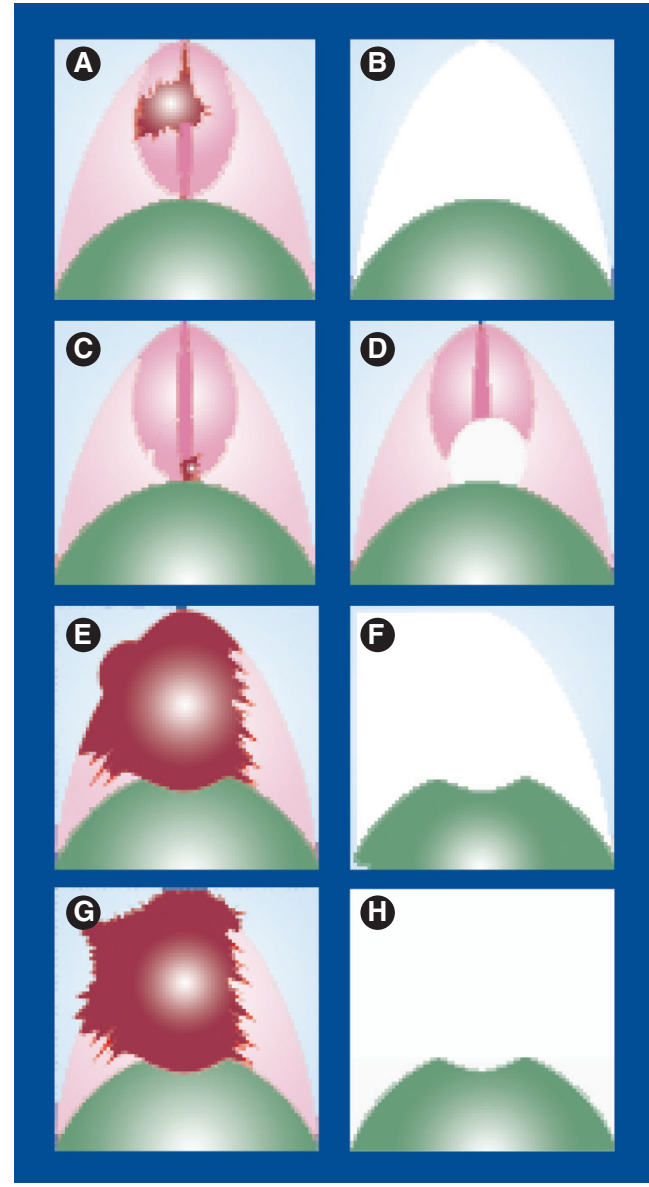

Figure 5. Ontogenetic compartment resection. (A \& B) Total compartment resection. The tumor-bearing ontogenetic compartment (pink) is completely resected after exposing its intact borders. Nonpermissive adjacent compartments of different embryonic origin (light blue and green) are retained despite narrow tumor margins. (C \& D) Modification of compartment resection as subcompartment resection for very early tumors. Within the ontogenetic compartment, tumor margins must be wide. At the compartment border, the inclusion of the intact bordering zone defines radical resection irrespective of margin width. (E \& F) Supracompartment resection for tumors beginning to transgress into a neighboring compartment of different embryonic origin. Total compartment resection is extended by wide excision of a part of the adjacent compartment at the site of inflammatory/fibrotic adherence. (G \& H) Multicompartment resection for overtly transgressing tumors. En bloc resection of two ontogenetic compartments. Also see legends for Figures 1 \& 3 .

\section{Ontogenetic compartment resection}

The ontogenetic compartment theory sets up a new principle of radicality for surgical tumor treatment, namely the resection of the tumorbearing compartment at its intact borders irrespective of the margin width. Nonlymphatic adjacent tissues of different ontogenetic compartments can safely be retained despite their close proximity to the tumor front. Figure 5 schematically sketches the principle of ontogenetic compartment resection, which should result, on the one hand, in maximal local control without adjuvant radiation and, on the other hand, in minimal treatment-related morbidity. Compartment resection can be modified to intracompartmental wide resection for very early tumors. Supracompartment as well as multicompartment resection may be treatment options for locally advanced cancer.

Clinical data for carcinoma of the uterine cervix [8,9], vagina [12], vulva [11] and rectum [1517] illustrate the great potential of ontogenetic compartment resection to improve local tumor control, reduce treatment-related morbidity and enhance overall survival. Results from more than 4000 patients with rectal cancer, treated with total mesorectal excision, demonstrated a $20 \%$ increase in overall survival compared with conventional surgery [16]. A similar survival benefit was shown with a monocentric trial for patients with cervical cancer undergoing total mesometrial resection [9].

\section{Future perspective}

It is hypothesized that the ontogenetic compartment theory of local tumor spread is generally valid and applicable for solid invasive tumors. So far, the theory is self-consistent and falsifiable by clinical testing. It can be tested for:

- The logic of argumentation

- Compliance with clinical facts

- The verification of hypothesis-derived predictions in clinical trials

A pre-requisite for testing is the knowledge of descriptive human embryology to identify the topography of the primordia (anlagen) for the region of interest. These must be followed during further development to delineate the ontogenetic compartments resulting as morphogenetic units from the differentiation and maturation of the corresponding anlagen.

The theory may be tested for any solid invasive tumor by relating the local tumor growth pattern, as depicted from its natural course, to the ontogenetic compartments deduced from the corresponding anlagen. A second testing scenario can be a comparison between the topography of the tissues resected with conventional tumor surgery and the specific ontogenetic compartment for the cancer of interest. If the 
conventional resection contains the complete ontogenetic compartment, local recurrence rates should approach zero, practically less than $5 \%$, for histopathologically proven $\mathrm{R} 0$ resection without adjuvant radiation.

A third testing scenario addresses the topography of local recurrences of the tumor of interest. It should be clarified whether their relapse sites correspond to remnants of the ontogenetic compartment retained with conventional tumor resection.

Finally, standard techniques of ontogenetic compartment resection have to be compared with the corresponding conventional surgical treatment by prospective multicenter trials. Ontogenetic compartment resection should reduce the rate of local recurrences to $5 \%$ or less at significantly decreased treatment-related morbidity. From previous experience, a relative increase of overall survival by up to $20 \%$ can be expected depending on the therapeutic significance of local tumor control.

From the prognostic analysis of lymph node metastasis assessed in more than 300 patients with cervical cancer, we found that the pattern of regional spread of this tumor entity can be comprehended and predicted from ontogenetic lymphatic compartments. Lymph node dissection based on ontogenetic anatomy achieved excellent regional tumor control without adjuvant radiation [Höckel M et al., Unpublished Data].
Whether the ontogenetic compartment theory opens up insights into the pattern of distant tumor spread as well remains to be investigated. Some steps of the metastatic cascade (e.g., homing, survival and tumor formation of circulating cancer cells in the target tissue) may depend on ontogenetic kinship between the donor and recipient compartments. Ontogenetic compartment resection holds the potential to significantly increase survival and decrease morbidity of cancer patients.

\section{Acknowledegements}

The author is indebted to JA Käs, Director of the Department of Physics at the University of Leipzig, for valuable comments. The artwork was drawn by N Lechenbauer, Ehenbichl, Austria.

\section{Financial \& competing interests disclosure}

Financial support was provided by the University of Leipzig, Germany, and the Leipzig School of Radical Pelvic Surgery. The funding source had no influence on the conduct of the research, the preparation of the article and in the decision to submit the paper for publication. The author has no other relevant affiliations or financial involvement with any organization or entity with a financial interest in or financial conflict with the subject matter or materials discussed in the manuscript apart from those disclosed.

No writing assistance was utilized in the production of this manuscript.

\section{Executive summary}

\section{Current principles \& practice of cancer surgery}

- Local tumor spread is traditionally regarded as an isotropic process of tissue infiltration irrespective of anatomic boundaries.

- Surgical practice derived from this view is wide tumor excision (i.e., resection of the malignant lesion with a metrically defined radial margin of microscopically tumor-free tissue).

- Conceptual inconsistencies and significant treatment failures despite adequate surgical performance question the present state and call for reconsideration.

\section{Ontogenetic anatomy}

- Ontogenetic anatomy describes and maps tissue compartments defined by embryonic development.

- It differs significantly from traditional clinical anatomy, which investigates structure as a tool to comprehend function.

Theory of tumor permeation within ontogenetic compartments

- The ontogenetic compartment theory accurately describes and predicts the pattern of local tumor permeation for all cancers investigated so far.

- It consistently explains the weaknesses of conventional cancer surgery.

\section{Ontogenetic compartment resection}

- Ontogenetic compartment resection, the application of ontogenetic instead of functional anatomy to guide cancer surgery achieved excellent local control rates with all tumor entities treated so far.

\section{Future perspective}

- The ontogenetic compartment theory is self-consistent and falsifiable by clinical testing. There is no argument against a general validity of the ontogenetic compartment theory for solid malignant tumors.

- Its clinical translation into compartment resection as new surgical treatment principle holds a great potential to improve local tumor control at decreased morbidity. 


\section{References}

Papers of special note have been highlighted as:

- of interest

". of considerable interest

1. Höckel M, Dornhöfer N. The hydra phenomenon of cancer: why tumors recur locally after microscopically complete resection. Cancer Res. 65, 2997-3002 (2005).

2. Rotman M, Sedlis A, Piedmonte MR et al. A Phase 3 randomized trial of postoperative pelvic irradiation in Stage 1B cervical carcinoma with poor prognostic features: follow-up of a gynecologic oncology group study. Int. J. Radiat. Oncol. Biol. Phys. 65, 169-176 (2006).

3. The ASTEC/EN.5 writing committee on behalf of the ASTEC/EN.5 study group. Adjuvant external beam radiotherapy in the treatment of endometrial cancer (MRC ASTEC and NCIC CTG EN.5 randomised trials): pooled trial results, systematic review, and meta-analysis. Lancet 373, 137-146 (2009).

4. Antonini N, Jones H, Horiot JC et al. Effect of age and radiation dose on local control after breast conserving treatment: EORTC trial 22881-10882. Radiother. Oncol. 82, 265-271 (2007).

5. Landoni F, Maneo A, Cormio G et al. Class 2 versus class 3 radical hysterectomy in stage 1B-2A cervical cancer: a prospective randomized study. Gynecol. Oncol. 80, 3-12 (2001).

6. Heaps JM, Fu YS, Montz FJ, Hacker NF, Berek JS. Surgical-pathologic variables predictive of local recurrence in squamous cell carcinoma of the vulva. Gynecol. Oncol. 38, 309-314 (1990).

7. Groenen S, Timmers PJ, Burger CW. Recurrence rate in vulvar carcinoma in relation to pathological margin distance. Int J. Gynecol. Cancer 20, 869-873 (2010).

8. Höckel M, Horn LC, Fritsch H. Association between the mesenchymal compartment of uterovaginal organogenesis and local tumour spread in stage $1 \mathrm{~B}-2 \mathrm{~B}$ cervical carcinoma: a prospective study. Lancet Oncol. 6, 751-756 (2005).

- First presentation of the ontogenetic compartment theory of local tumor spread supported by the results of a prospective monocentric trial of compartment resection for cervical cancer.

9. Höckel M, Horn LC, Manthey N et al. Resection of the embryologically defined uterovaginal (Müllerian) compartment and pelvic control in patients with cervical cancer: a prospective analysis. Lancet Oncol. 10, 683-692 (2009).

- First demonstration with cervical cancer that local tumor recurrences arise from compartment remnants remaining in situ after conventional radical surgery.

10. Höckel M, Kahn T, Einenkel J et al. Local spread of cervical cancer revisited. A clinical and pathological pattern analysis. Gynecol. Oncol. 117, 401-408 (2010).

11. Höckel M, Schmidt K, Bornmann K, Horn LC, Dornhöfer N. Vulvar field resection: Novel approach to the surgical treatment of vulvar cancer based on ontogenetic anatomy. Gynecol. Oncol. 119, 106-113 (2010).

- Initial promising results from a prospective monocentric study of compartment resection for vulvar cancer.

12. Höckel M, Horn LC, Illig R, Dornhöfer N, Fritsch H. Ontogenetic anatomy of the distal vagina: Relevance for local tumor spread and implications for cancer surgery. Gynecol. Oncol. 122, 313-318 (2011).

- First proof of local tumor permeation within ontogenetic compartments for carcinoma involving the distal vagina.

13. Garcia-Bellido A, Ripoll P, Morata G. Developmental compartmentalization of the wing disk of Drosophila. Nature 245, 251-253 (1973).

- First proof of developmental compartments maintained through differentiation and maturation.
14. Dahmann C, Oates AC, Brand M. Boundary formation and maintenance in tissue development. Nat. Rev. 12, 43-55 (2011).

- Comprehensive state-of-the-art review on developmental compartments.

15. Heald RJ, Ryan RDH. Recurrence and survival after total mesorectal excision for rectal cancer. Lancet 327, 1479-1482 (1986).

- First successful application of ontogenetic anatomy to guide cancer surgery with total mesorectal excision for carcinoma of the rectum.

16. Quirke P, Dixon MF, Durdey P, Williams NS. Local recurrence of rectal adenocarcinoma due to inadequate surgical resection. Lancet 328, 996-998 (1986).

- Systematic pathoanatomical analysis of local failures in the conventional surgical treatment of rectal cancer.

17. Rullier E, Laurent C, Bretagnol F, Rullier A, Vendrely V, Zerbib F. Sphincter-saving resection for all rectal carcinomas. The end of the 2-cm distal rule. Ann. Surg. 241, 465-469 (2005).

18. Darakhshan A, Lin BPFC, Chan C, Chapuis PH, Dent OF, Bokey L. Correlates and outcomes of tumor adherence in resected colonic and rectal cancers. Ann. Surg. 247, 650-658 (2008).

19. Mannino M, Yarnold J. Effect of breast-duct anatomy and wound-healing responses on local tumour recurrence after primary surgery for early breast cancer. Lancet Oncol. 10, 425-429 (2009).

20. Höckel M, Dornhöfer N. Understanding and preventing local tumour recurrence. Lancet Oncol. 10, 645-646 (2009).

21. Makino I, Kitagawa H, Ohta $\mathrm{T}$ et al. Nerve plexus invasion in pancreatic cancer. Pancreas 37, 358-365 (2008).

22. Kalani MYS, Kalani MA, Gwinn R, Keogh B, Tse VCK. Embryological development of the human insula and its implications for the spread and resection of insular gliomas. Neurosurg. Focus 27, E2 (2009). 